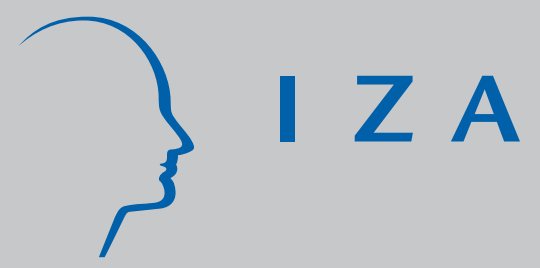

IZA DP No. 74

Strategic Mismatches in Competing Teams

Matthias Kräkel

November 1999 


\title{
Strategic Mismatches in Competing Teams
}

\author{
Matthias Kräkel \\ University of Bonn, Germany
}
Discussion Paper No. 74 December 1999

\author{
IZA \\ P.O. Box 7240 \\ D-53072 Bonn \\ Germany \\ Tel.: +49-228-3894-0 \\ Fax: +49-228-3894-210 \\ Email: iza@iza.org
}

This Discussion Paper is issued within the framework of IZA's research area General Labor Economics. Any opinions expressed here are those of the author(s) and not those of the institute. Research disseminated by IZA may include views on policy, but the institute itself takes no institutional policy positions.

The Institute for the Study of Labor (IZA) in Bonn is a local and virtual international research center and a place of communication between science, politics and business. IZA is an independent, nonprofit limited liability company (Gesellschaft mit beschränkter Haftung) supported by the Deutsche Post AG. The center is associated with the University of Bonn and offers a stimulating research environment through its research networks, research support, and visitors and doctoral programs. IZA engages in (i) original and internationally competitive research in all fields of labor economics, (ii) development of policy concepts, and (iii) dissemination of research results and concepts to the interested public. The current research program deals with (1) mobility and flexibility of labor markets, (2) internationalization of labor markets and European integration, (3) the welfare state and labor markets, (4) labor markets in transition, (5) the future of work, (6) project evaluation and (7) general labor economics.

IZA Discussion Papers often represent preliminary work and are circulated to encourage discussion. Citation of such a paper should account for its provisional character. 
IZA Discussion Paper No. 74

December 1999

\section{ABSTRACT}

\section{Strategic Mismatches in Competing Teams}

This paper discusses the strategic role of mismatching, where players voluntarily form inefficient teams or forego the formation of efficient teams, respectively. Strategic mismatching can be rational when players realize a competitive advantage (e.g. harming other competitors). In addition, the results show that free riding can be beneficial for a team in combination with strategic mismatching and that the loser's curse may be welfare improving by mitigating the problem of strategic mismatching.

JEL Classification: C72, D21, J41, J44

Keywords: Economies of scope, free-rider effect, mismatch, teams, tournament

Matthias Kräkel

Department of Economics

BWL II

University of Bonn

Adenauerallee 24-42

D-53113 Bonn

Germany

Tel.: +49228739211

Fax: +49228739210

Email: m.kraekel@uni-bonn.de

* I would like to thank Silke Becker and Gunter Steiner for helpful comments. 


\section{Introduction}

On July 31, 1993, an interesting transaction was sealed within American professional baseball. On that day, the Blue Jays acquired Rickey Henderson, "the best lead-off hitter in the history of baseball" (O'Malley and O'Malley 1994, p. 11). This deal seems to be puzzling, because the Blue Jays needed a pitcher and not a hitter at that time. However, the acquisition of Henderson was quite rational for the Blue Jays since it successfully prevented that the Yankees or other competitors were able to acquire Henderson for strengthening their teams. We can also imagine other examples of strategic mismatching. For example, a law firm hires the best law students that have passed their exams in a certain year not because additional lawyers are needed in the firm at that time but because thereby other law firms are not able to realize a competitive advantage by hiring these students. Or a firm tries to raid a competing firm by acquiring some of its managers despite of their large amount of firm-specific human capital solely to harm the competing firm.

This paper discusses these and other types of strategic mismatches, and the conditions under which strategic mismatching is possible. In general, strategic mismatching is given when players voluntarily form inefficient teams or forego the formation of efficient teams, respectively. ${ }^{1}$ There are several effects which influence the possibility of a strategic mismatch: the well-known free-rider effect in teams, a cost effect based on economies or diseconomies of scope generated by the team composition, a winner's curse and a loser's curse resulting from overestimating or underestimating the gains from team formation, respectively, and the influence of luck in the final tournament

\footnotetext{
${ }^{1}$ The terms "matching" and "mismatching" come from labor economics and denote the formation of efficient or inefficient combinations of employers and workers, respectively. See, e.g., Jovanovic (1984), Mortensen (1988).
} 
competition between the one-player and/or two-players teams. The model considers two incumbent players who one after another can offer a team contract (i.e. an equal sharing arrangement) to a new entrant into the market. If no (two-players) team is formed, the two incumbents and the entrant will compete as single players against each other in a tournament at the final stage of the game. If one of the incumbents forms a team with the entrant there will be a tournament competition between a two-players team (consisting of the one incumbent and the entrant) and a one-player team (consisting of the other incumbent). This model is best reflected by the market for professional services (e.g. the market for lawyers) where a new entrant (e.g. a new lawyer) can either work as a self-employed or form a partnership (e.g. a law firm) with one of the incumbents. Several interesting results can be derived for this model. For example, when luck plays a dominant role in the tournament, the marginal costs of effort are large, and the winner prize is small, strategic mismatching can be rational where players choose the organizational form - self-employment or team - that minimizes the incentives to exert effort. In this context, diseconomies of scope and free riding can be beneficial for the team to reduce work incentives. Another result shows that the loser's curse, a kind of decision anomaly, may be welfare improving by mitigating the perils of strategic mismatching.

The paper is organized as follows. Section 2 describes the model and solves the tournament competition at the final stage of the sequential game. Section 3 contains the main results of the paper. In this section, three types of mismatches are defined and the conditions under which these mismatches may exist are analyzed. The last section concludes. 


\section{The Model}

In the following model, three risk neutral players compete for a benefit $B$ (e.g., a large market share or highly profitable order). The three players are the two incumbents $I_{1}$ and $I_{2}$ and the new entrant $E$ into the market. Before competition starts there is a sequential contracting process which is described by Figure 1 .

\section{[Figure 1]}

In $t_{1}$, incumbent $I_{1}$ can offer a team contract to the entrant $E$ (e.g., the three players belong to the professional services and $I_{1}$ offers $E$ a partnership contract). If $I_{1}$ offers a contract and $E$ accepts, the game will continue in $t_{5}$, where the team consisting of $I_{1}$ and $E$ competes against $I_{2}$ in a simple tournament. The winner of the tournament - the team $\left\{I_{1}, E\right\}$ or $I_{2}-$ receives the benefit $B(B>0)$, whereas the loser gets nothing. ${ }^{2}$ If $I_{1}$ does not offer a contract to $E$ or $E$ rejects $I_{1}$ 's offer in $t_{2}$, the incumbent $I_{2}$ has to decide about a contract offer. If $I_{2}$ offers a team contract and $E$ agrees to it, the team $\left\{I_{2}, E\right\}$ will compete against $I_{1}$ in $t_{5}$. If $I_{2}$ chooses not to offer a contract or $E$ rejects $I_{2}$ 's offer in $t_{4}$, there will be a standard tournament between the three single players $I_{1}, I_{2}$, and $E$ in $t_{5}$. Before I start to discuss possible mismatches that can arise during $t_{1}-t_{4}$, further details about the tournament subgame in $t_{5}$ have to be described.

There are three possible states in $t_{5}$. First, no team $\left\{I_{1}, E\right\}$ or $\left\{I_{2}, E\right\}$, respectively, has been formed. This state is denoted by $s_{1}$. In this state,

\footnotetext{
${ }^{2}$ As an alternative, we can choose a winner prize $B_{1}$ and a loser prize $B_{2}\left(<B_{1}\right)$ for the tournament. But this modification would not be of great consequence, because only the prize spread $B_{1}-B_{2}(=: B)$ generates incentives in the tournament and not the absolute values of the winner and the loser prizes.
} 
the three players are independent competitors. Each player $i\left(i=I_{1}, I_{2}, E\right)$ has a simple linear production function $q_{i}=e_{i}+\varepsilon_{i}$ where $e_{i}$ denotes $i$ 's effort and $\varepsilon_{i}$ a random or luck component. ${ }^{3}$ The $\varepsilon_{i}$ s are assumed to be independently and identically distributed (i.i.d.-assumption) according to a cumulative distribution function $F(\varepsilon)$ with density $f(\varepsilon)$. For simplicity, we assume that the $\varepsilon_{i} \mathrm{~s}$ are uniformly distributed over the interval $[0, \bar{\varepsilon}]$ with $F(\varepsilon)=\varepsilon / \bar{\varepsilon}$ and $f(\varepsilon)=1 / \bar{\varepsilon}^{4}$ The player with the highest realized outcome $q_{i}$ will be the winner of the tournament and receives the benefit $B$. Player $i$ 's disutility of effort (in monetary terms) is described by the cost function $c\left(e_{i}\right)=\frac{k}{2} e_{i}^{2}(k>0)$. Each competitor wants to maximize his expected utility which is identical with his expected net income:

$$
E U_{i}\left(e_{i} ; s_{1}\right)=B \cdot \operatorname{pr}\{i \text { wins }\}-\frac{k}{2} e_{i}^{2} .
$$

The analysis is restricted to symmetric equilibria in the tournament subgame, where each player chooses the same amount of effort $e^{*}=$ $e_{I_{1}}^{*}=e_{I_{2}}^{*}=e_{E}^{*}{ }^{5} \quad$ Thus, the winning probability $\operatorname{pr}\{i$ wins $\}$ can be written as $\operatorname{pr}\{i$ wins $\}=\operatorname{pr}\left\{q_{i}>q_{(2)}\right\}=\operatorname{pr}\left\{e_{i}+\varepsilon_{i}>e^{*}+\varepsilon_{(2)}\right\}=$ $\operatorname{pr}\left\{\varepsilon_{(2)}-\varepsilon_{i}<e_{i}-e^{*}\right\}=\operatorname{pr}\left\{X<e_{i}-e^{*}\right\}=G\left(e_{i}-e^{*}\right)$ where both $q_{(2)}$ and

\footnotetext{
${ }^{3}$ Most of the assumptions follow the standard tournament model by Lazear and Rosen (1981).

${ }^{4}$ In the following, we have to calculate with order statistics to derive the equilibrium efforts for the tournament subgame. It is well-known that calculating with order statistics implies some difficulties. Therefore, the simplifying assumption of uniformly distributed luck is used in this model. Note also that the paper will focus on mismatching and not on tournament competition.

${ }^{5}$ This restrictive assumption is not unusual in the tournament literature; see, for example, Nalebuff and Stiglitz (1983, pp. 26-27), Lazear (1989, p. 565). There may be asymmetric equilibria, too. But intuitively it is reasonable to think of symmetric equilibria, because the three players have identical characteristics.
} 
$\varepsilon_{(2)}$ denote the highest of two order statistics, respectively, ${ }^{6}$ and $X:=\varepsilon_{(2)}-\varepsilon_{i}$ with cumulative distribution function $G(x)$ and density $g(x)$. Therefore, equation (1) can be rewritten as

$$
E U_{i}\left(e_{i} ; s_{1}\right)=B \cdot G\left(e_{i}-e^{*}\right)-\frac{k}{2} e_{i}^{2}
$$

The first order condition $E U_{i}^{\prime}=B \cdot g\left(e^{*}-e_{i}\right)-k e_{i}=B \cdot g(0)-k e_{i}=0$ gives the Nash equilibrium effort

$$
e_{i}^{*}=e^{*}=\frac{B g(0)}{k}=\frac{B}{k \bar{\varepsilon}}, \quad i=I_{1}, I_{2}, E
$$

where the last expression follows from the assumption of uniformly distributed luck. ${ }^{7}$ By substituting (3) into (2) we obtain ${ }^{8}$

$$
E U_{i}\left(e_{i}^{*} ; s_{1}\right)=\frac{B}{3}-\frac{B^{2}}{2 k \bar{\varepsilon}^{2}}, \quad i=I_{1}, I_{2}, E .
$$

The second state, $s_{2}$, considers the case where $I_{2}$ and $E$ form a team $\left\{I_{2}, E\right\}$, which competes against $I_{1}$ in the tournament. It is assumed that $I_{2}$ and $E$ produce an outcome according to a linear team production function $q\left(e_{I_{2}}, e_{E}\right)=e_{I_{2}}+e_{E}+\varepsilon_{I_{2}}$. Here, $\varepsilon_{I_{2}}$ denotes the same stochastic luck as in state $s_{1} \cdot{ }^{9}$ The team effect of joint production is reflected by the cost function

\footnotetext{
${ }^{6}$ I.e., $q_{(2)}=\max \left\{q_{m}, q_{n}\right\}$ and $\varepsilon_{(2)}=\max \left\{\varepsilon_{m}, \varepsilon_{n}\right\}$ with $m, n \in\left\{I_{1}, I_{2}, E\right\} \backslash\{i\}$ and $m \neq n$.

${ }^{7}$ See the Appendix A for the derivation of the density $g(x)$. The second order condition holds for uniformly distributed $\varepsilon_{i}$.

${ }^{8}$ Note that $G(0)=1 / 3$.

${ }^{9}$ Alternatively, we can define a team production function $q\left(e_{I_{2}}, e_{E}\right)=e_{I_{2}}+e_{E}+\varepsilon_{I_{2}}+\varepsilon_{E}$, but then there is an additional advantage of forming a team, because the realizations of the $\varepsilon_{i} \mathrm{~s}$ are exclusively nonnegative. This additional effect would bias the trade-off considered in this paper and should therefore be eliminated. As another alternative, we can assume that there is positive luck as well as negative luck, i.e. the $\varepsilon_{i}$ s are distributed over $[-\bar{\varepsilon}, \bar{\varepsilon}]$. But then the variance of the team production function $q\left(e_{I_{2}}, e_{E}\right)=e_{I_{2}}+e_{E}+\varepsilon_{I_{2}}+\varepsilon_{E}$ is
} 
of each team member:

$$
c\left(e_{i}\right)=\frac{k}{2 \gamma_{I_{2}}} e_{i}^{2}, \quad \gamma_{I_{2}}>0, \quad i=I_{2}, E .
$$

The new cost parameter $\gamma_{I_{2}}$ can be either greater or lower than one, where $\gamma_{I_{2}}>1$ indicates economies of scope and $\gamma_{I_{2}}<1$ diseconomies of scope, respectively. Furthermore, we assume that if the team wins the benefit $B$ will be shared equally among $I_{2}$ and $E .^{10}$ Thus, $I_{2}$ 's and $E$ 's expected utility in state $s_{2}$ is given by

$$
E U_{i}\left(e_{i} ; s_{2}\right)=\frac{B}{2} \cdot \operatorname{pr}\left\{\text { team }\left\{I_{2}, E\right\} \text { wins }\right\}-\frac{k}{2 \gamma_{I_{2}}} e_{i}^{2}, \quad i=I_{2}, E .
$$

Incumbent $I_{1}$ remains alone. It is assumed that he has the same production function and the same cost function as in state $s_{2}$. Therefore, his expected utility is

$$
E U_{I_{1}}\left(e_{I_{1}} ; s_{2}\right)=B \cdot \operatorname{pr}\left\{I_{1} \text { wins }\right\}-\frac{k}{2} e_{I_{1}}^{2}
$$

Comparing equations (6) and (7) we see that a trade-off has to be taken into account by $I_{2}$ and $E$ when they decide about forming a team: Economies of scope (i.e., $\gamma_{I_{2}}>1$ ) can make the formation of a team attractive, because $I_{2}$ and $E$ would realize lower marginal costs. This effect is defined as cost effect. On the other hand, $I_{2}$ and $E$ will only receive half of the benefit $B$ if they win against $I_{1}$. This effect twice as high as the variance of a single competitor's production function. This additional effect should be eliminated, too, because this paper focuses on other aspects, which become more clear when the underlying stochastic structure is as simple as possible.

${ }^{10}$ Of course, such an equal sharing arrangement is not always optimal. It is a simplifying assumption, which can be motivated by the fact that individual contributions to the joint team outcomes are often non-contractible. Therefore, the team members agree on an equal sharing contract. In addition, the equal sharing of $B$ can be characterized as the outcome of the Nash bargaining solution. 
reduces the team members' incentives to exert effort and is well-known as free-rider effect. The winning probability $\operatorname{pr}\left\{\operatorname{team}\left\{I_{2}, E\right\}\right.$ wins $\}$ can be written as $\operatorname{pr}\left\{\right.$ team $\left\{I_{2}, E\right\}$ wins $\}=\operatorname{pr}\left\{e_{I_{2}}+e_{E}+\varepsilon_{I_{2}}>e_{I_{1}}+\varepsilon_{I_{1}}\right\}=$ $\operatorname{pr}\left\{\varepsilon_{I_{1}}-\varepsilon_{I_{2}}<e_{I_{2}}+e_{E}-e_{I_{1}}\right\}=\operatorname{pr}\left\{Y<e_{I_{2}}+e_{E}-e_{I_{1}}\right\}=H\left(e_{I_{2}}+e_{E}-e_{I_{1}}\right)$ where $Y:=\varepsilon_{I_{1}}-\varepsilon_{I_{2}}$ has a cumulative distribution function $H(y)$ and a density $h(y)$. Now, equations (6) and (7) can be written as

$$
\begin{gathered}
E U_{I_{2}}\left(e_{I_{2}} ; s_{2}\right)=\frac{B}{2} \cdot H\left(e_{I_{2}}+e_{E}-e_{I_{1}}\right)-\frac{k}{2 \gamma_{I_{2}}} e_{I_{2}}^{2} \\
E U_{E}\left(e_{E} ; s_{2}\right)=\frac{B}{2} \cdot H\left(e_{I_{2}}+e_{E}-e_{I_{1}}\right)-\frac{k}{2 \gamma_{I_{2}}} e_{E}^{2} \\
E U_{I_{1}}\left(e_{I_{1}} ; s_{2}\right)=B \cdot\left[1-H\left(e_{I_{2}}+e_{E}-e_{I_{1}}\right)\right]-\frac{k}{2} e_{I_{1}}^{2} .
\end{gathered}
$$

From the first order conditions we obtain the following reaction functions: ${ }^{11}$

$$
\begin{gathered}
\frac{B}{2} h\left(e_{I_{2}}+e_{E}-e_{I_{1}}\right)-\frac{k}{\gamma_{I_{2}}} e_{I_{2}}=0 \\
\frac{B}{2} h\left(e_{I_{2}}+e_{E}-e_{I_{1}}\right)-\frac{k}{\gamma_{I_{2}}} e_{E}=0 \\
B h\left(e_{I_{2}}+e_{E}-e_{I_{1}}\right)-k e_{I_{1}}=0 .
\end{gathered}
$$

Equations (11) and (12) show that if a Nash equilibrium exists, the team members $I_{2}$ and $E$ will exert the same amount of effort, i.e. $e_{I_{2}}=e_{E}$. Combining this result with (11) (or (12)) and (13) yields the following

\footnotetext{
${ }^{11}$ For the derivation of $h(y)$ see Appendix A. We assume that the second order conditions hold and a Nash equilibrium exists. The problem that this additional assumption is needed is already known in the tournament literature. See, e.g., Lazear and Rosen (1981, p. 845, fn. 2); Nalebuff and Stiglitz (1983, p. 29); Lazear (1989, p. 565, fn. 3).
} 
condition, which emphasizes the trade-off between the cost effect and the free-rider effect:

$$
e_{E}=e_{I_{2}}=\frac{\gamma_{I_{2}}}{2} e_{I_{1}}
$$

Equation (14) shows that whether a team member or $I_{1}$ exerts more effort in equilibrium depends on the relation between the cost effect and the free-rider effect. If the cost effect is dominant (i.e. $\gamma_{I_{2}}>2$ ), each team member will exert more effort than $I_{1}$. If the free-rider effect is dominant (i.e. $2>\gamma_{I_{2}}$ ), the opposite will hold.

To derive the equilibrium efforts we have to substitute the concrete form of the density $h(y)$ into (11)-(13). The results of Appendix A show that the convolution $h(y)$ of two uniform (or rectangular) densities is a triangular density function, which is symmetric around zero. Thus, we have to discuss two different cases. First, it is possible that $e_{I_{2}}+e_{E} \leq e_{I_{1}}$. In that case, we have to use the left-hand part of the triangular density $h(y)$ for solving (11)-(13). From (14) we know that this scenario will become possible, if and only if $\gamma_{I_{2}} \leq 1$, i.e. if there are diseconomies of scope or a "negative" cost effect. ${ }^{12}$ Secondly, the opposite relation $e_{I_{2}}+e_{E}>e_{I_{1}}$ can hold. In this case, the right-hand part of the density $h(y)$ becomes relevant and we have economies of scope or a "positive" cost effect for the team $\left\{I_{2}, E\right\}$, i.e. $\gamma_{I_{2}}>1$. After some calculations the "negative scenario" $e_{I_{2}}+e_{E} \leq e_{I_{1}}$ yields the following expressions for the equilibrium efforts: ${ }^{13}$

$$
e_{I_{2}}^{*}=e_{E}^{*}=\frac{\gamma_{I_{2}}}{2} \frac{B \bar{\varepsilon}}{B-B \gamma_{I_{2}}+k \bar{\varepsilon}^{2}}
$$

\footnotetext{
${ }^{12}$ Because of (14) the condition $e_{I_{2}}+e_{E} \leq e_{I_{1}}$ can be rewritten as $2 e_{E} \leq e_{I_{1}}$ $\Longleftrightarrow 2 \frac{\gamma_{I_{2}}}{2} e_{I_{1}} \leq e_{I_{1}} \Longleftrightarrow \gamma_{I_{2}} \leq 1$ assuming that no negative effort levels are possible.

${ }^{13}$ Note that the three effort levels are positive, because $\gamma_{I_{2}} \leq 1$ in this "negative scenario".
} 


$$
e_{I_{1}}^{*}=\frac{B \bar{\varepsilon}}{B-B \gamma_{I_{2}}+k \bar{\varepsilon}^{2}} .
$$

A comparison of (15) and (16) shows the trade-off between the cost effect and the free-rider effect (in analogy to (14)). In addition, we see that the strategic interaction between the team $\left\{I_{2}, E\right\}$ and $I_{1}$ in the tournament results in a spillover of the cost effect. An increasing $\gamma_{I_{2}}$ reduces the denominator of (15) as well as the denominator of (16) and results in increasing efforts of all players. However, the cost effect is larger for the two team members, because an increasing $\gamma_{I_{2}}$ additionally increases the numerator of (15). Substituting (15) and (16) into (8)-(10) and using the concrete form of the distribution function $H(y)$ (see Appendix A) gives the expected utilities in equilibrium: ${ }^{14}$

$$
\begin{gathered}
E U_{I_{2}}^{-}\left(e_{I_{2}}^{*} ; s_{2}\right)=E U_{E}^{-}\left(e_{E}^{*} ; s_{2}\right)=\frac{1}{8} B k \bar{\varepsilon}^{2} \frac{2 k \bar{\varepsilon}^{2}-B \gamma_{I_{2}}}{\left(B-B \gamma_{I_{2}}+k \bar{\varepsilon}^{2}\right)^{2}} \\
E U_{I_{1}}^{-}\left(e_{I_{1}}^{*} ; s_{2}\right)=B \frac{B^{2}\left(1-\gamma_{I_{2}}\right)^{2}+B k \bar{\varepsilon}^{2}\left(\frac{3}{2}-2 \gamma_{I_{2}}\right)+\frac{1}{2} k^{2} \bar{\varepsilon}^{4}}{\left(B-B \gamma_{I_{2}}+k \bar{\varepsilon}^{2}\right)^{2}} .
\end{gathered}
$$

Similar calculations can be made for the "positive scenario". Now we obtain

$$
\begin{gathered}
e_{I_{2}}^{*}=e_{E}^{*}=\frac{\gamma_{I_{2}}}{2} \frac{B \bar{\varepsilon}}{B \gamma_{I_{2}}-B+k \bar{\varepsilon}^{2}} \\
e_{I_{1}}^{*}=\frac{B \bar{\varepsilon}}{B \gamma_{I_{2}}-B+k \bar{\varepsilon}^{2}}
\end{gathered}
$$

as equilibrium efforts and

$$
\begin{aligned}
& E U_{I_{2}}^{+}\left(e_{I_{2}}^{*} ; s_{2}\right)= E U_{E}^{+}\left(e_{E}^{*} ; s_{2}\right)= \\
& \frac{B}{8} \frac{4 B^{2}\left(1-\gamma_{I_{2}}\right)^{2}+B k \bar{\varepsilon}^{2}\left(7 \gamma_{I_{2}}-8\right)+2 k^{2} \bar{\varepsilon}^{4}}{\left(B \gamma_{I_{2}}-B+k \bar{\varepsilon}^{2}\right)^{2}} \\
& E U_{I_{1}}^{+}\left(e_{I_{2}}^{*} ; s_{2}\right)=\frac{1}{2} B k \bar{\varepsilon}^{2} \frac{k \bar{\varepsilon}^{2}-B}{\left(B \gamma_{I_{2}}-B+k \bar{\varepsilon}^{2}\right)^{2}}
\end{aligned}
$$

\footnotetext{
${ }^{14}$ The "_" indicates that here the "negative scenario" is considered.
} 
as expected utilities in equilibrium. ${ }^{15}$

The third state, $s_{3}$, considers the case where $I_{1}$ and $E$ form a team $\left\{I_{1}, E\right\}$ in $t_{2}$ which competes against $I_{2}$ in the tournament in $t_{5}$. The above considerations concerning state $s_{2}$ analogously hold for $s_{3}$. Interchanging the indices $I_{2}$ and $I_{1}$ in $(15)-(22)$ and substituting $s_{3}$ for $s_{2}$ yields

$$
\begin{gathered}
E U_{I_{1}}^{-}\left(e_{I_{1}}^{*} ; s_{3}\right)=E U_{E}^{-}\left(e_{E}^{*} ; s_{3}\right)=\frac{1}{8} B k \bar{\varepsilon}^{2} \frac{2 k \bar{\varepsilon}^{2}-B \gamma_{I_{1}}}{\left(B-B \gamma_{I_{1}}+k \bar{\varepsilon}^{2}\right)^{2}} \\
E U_{I_{2}}^{-}\left(e_{I_{2}}^{*} ; s_{3}\right)=B \frac{B^{2}\left(1-\gamma_{I_{1}}\right)^{2}+B k \bar{\varepsilon}^{2}\left(\frac{3}{2}-2 \gamma_{I_{1}}\right)+\frac{1}{2} k^{2} \bar{\varepsilon}^{4}}{\left(B-B \gamma_{I_{1}}+k \bar{\varepsilon}^{2}\right)^{2}}
\end{gathered}
$$

for the expected utilities in the "negative scenario" equilibrium of state $s_{3}$ and

$$
\begin{aligned}
& E U_{I_{1}}^{+}\left(e_{I_{1}}^{*} ; s_{3}\right)= E U_{E}^{+}\left(e_{E}^{*} ; s_{3}\right)= \\
& \frac{B}{8} \frac{4 B^{2}\left(1-\gamma_{I_{1}}\right)^{2}+B k \bar{\varepsilon}^{2}\left(7 \gamma_{I_{1}}-8\right)+2 k^{2} \bar{\varepsilon}^{4}}{\left(B \gamma_{I_{1}}-B+k \bar{\varepsilon}^{2}\right)^{2}} \\
& E U_{I_{2}}^{+}\left(e_{I_{2}}^{*} ; s_{3}\right)=\frac{1}{2} B k \bar{\varepsilon}^{2} \frac{k \bar{\varepsilon}^{2}-B}{\left(B \gamma_{I_{1}}-B+k \bar{\varepsilon}^{2}\right)^{2}}
\end{aligned}
$$

for the expected utilities in the "positive scenario" equilibrium of state $s_{3}$ where $\gamma_{I_{1}}$ indicates the economies or diseconomies of scope when $I_{1}$ and $E$ form a team in analogy to $\gamma_{I_{2}}$ in state $s_{2} \cdot{ }^{16}$

The preliminary results of this section describe the equilibrium solutions of the tournament subgame in $t_{5}$ for the three states $s_{1}, s_{2}$, and $s_{3}$. The next section focuses on strategic mismatching that can arise during $t_{1}-t_{4}$. It contains the main results of the paper.

\footnotetext{
${ }^{15}$ Here, "+" indicates the "positive scenario". Note that $\gamma_{2}>1$ in the "positve scenario" guarantees positve efforts in equilibrium.

${ }^{16}$ I.e., $I_{1}$ 's and E's cost function can be described by $c\left(e_{i}\right)=\frac{k}{2 \gamma_{I_{1}}} e_{i}^{2}, \gamma_{I_{1}}>0, i=I_{1}$, $E$, in state $s_{3}$.
} 


\section{Strategic mismatching}

In this section, we will look for subgame perfect equilibria in the game described by Figure 1 that lead to (strategic) mismatching between $I_{1}, I_{2}$, and $E$. The problem of mismatching might become relevant during $t_{1}-t_{4}$, when the three players decide about forming a team. Nevertheless, the results of Section 2 are still important, because the players have to regard the tournament in $t_{5}$ and the three states $s_{1}, s_{2}$, and $s_{3}$ when deciding about a team formation. Thus, the objective to reach or to avoid a certain outcome in $t_{5}$ can be the major cause for strategic mismatching during $t_{1}-t_{4}$. Before discussing the possibility of strategic mismatching under different information structures, we have to differentiate between the various types of mismatches. To reduce complexity, the following analysis is restricted to mismatches that can arise in connection with player $I_{1}$. Similar results could be derived by focusing on player $I_{2}$. Concerning player $I_{1}$ there are three types of mismatch equilibria in the game:

- Mismatch I equilibrium: $I_{1}$ and $E$ form a team, although the team $\left\{I_{2}, E\right\}$ would generate larger economies of scope, i.e. $\gamma_{I_{2}}>\gamma_{I_{1}}>1$. Without $I_{1}$ 's offer in $t_{1}$ there would be an efficient match between $I_{2}$ and $E$.

- Mismatch II equilibrium: $I_{1}$ and $E$ form a team in spite of diseconomies of scope, i.e. $\gamma_{I_{2}}<\gamma_{I_{1}}<1$. Without $I_{1}$ 's offer in $t_{1}$ there would be efficient matching, where all players remain self-employed.

- Mismatch III equilibrium: $I_{1}$ and $E$ do not form a team despite of economies of scope, i.e. $\gamma_{I_{1}}, \gamma_{I_{2}}>1$. 
Mismatch I deals with the classical problem of mismatching where the wrong players decide to form a team. Mismatch II considers the case in which the players form a team when there should be no team because of efficiency reasons. Mismatch III considers the opposite case where players forego an efficient match. In the following subsections, the existence of these three types of mismatch equilibria will be discussed under different information structures. Subsection 3.1 discusses the symmetric information case of purely strategic mismatches. Subsection 3.2 adds asymmetric information to the problem of mismatching and assumes that the incumbents have less information about $\gamma_{I_{1}}$ and $\gamma_{I_{2}}$ than the entrant. Subsection 3.3 considers the case of better informed incumbents concerning $\gamma_{I_{1}}$ and $\gamma_{I_{2}}$.

\subsection{Symmetric Information}

Information problems are the traditional reason for mismatching in economies. In this subsection, information problems are completely excluded. Thus, we can concentrate on purely strategic mismatching, because all the mismatches left must be voluntary. Here, we assume that the cost parameters $\gamma_{I_{1}}$ and $\gamma_{I_{2}}$ are common knowledge in the whole game. By that means, each player is able to correctly calculate the consequences of the cost effect and the free-rider effect and therefore the possible outcomes in the states $s_{1}, s_{2}$, and $s_{3}$ in $t_{5}$. Under these assumptions we obtain the following results:

\section{Proposition 1}

If all players have complete information the following will hold:

(i) There does not exist a mismatch I equilibrium.

(ii) There is the possibility of a mismatch II equilibrium if and only if $3 B<2 k \bar{\varepsilon}^{2}$. 
(iii) There is the possibility of a mismatch III equilibrium if and only if $27 B^{2}+3 B k \bar{\varepsilon}^{2}<2 k^{2} \bar{\varepsilon}^{4}$.

\section{Proof. See Appendix B.}

The result of Proposition 1(i) seems to be plausible. In the case of symmetric information $E$ knows that forming a team with $I_{2}$ is better for him than forming a team with $I_{1}$ because of $\gamma_{I_{2}}>\gamma_{I_{1}}$. In $t_{2}$ player $E$ anticipates that he will receive a contract offer from $I_{2}$ in $t_{3}$. Therefore, he would never accept the less attractive offer from player $I_{1}$ in $t_{2}$. Hence, without any information problem a mismatch I equilibrium cannot exist. The result of Proposition 1(ii) seems to be puzzling. Here, $I_{1}$ and $E$ form a team in spite of the free-rider effect and diseconomies of scope, i.e. there is no cost effect, which can outweigh the disadvantages of the free-rider effect. Thus, we would expect the non-existence of a mismatch II equlibrium as a plausible result. Such expectations are supported by the fact that with Cournot competition instead of tournament competition in $t_{5}$ a mismatch II will not arise in a situation with symmetric information (see Kräkel 1999). Thus, the kind of competition in $t_{5}$ seems to be the cause for the result of Proposition 1(ii). Here, we have a tournament competition, which makes a mismatch II equilibrium possible as long as the parameter condition $3 B<2 k \bar{\varepsilon}^{2}$ is met. $^{17}$ This puzzling result can be explained by looking at the right side of the parameter condition. First, the condition means that luck plays a dominant role for the outcome of the tournament as $\bar{\varepsilon}$ is required to be large which implies a large variance for the stochastic luck component $\varepsilon_{i} \cdot{ }^{18}$ Then it is

\footnotetext{
${ }^{17}$ Note that this condition implies that the expected utility of each player has to be positive in state $s_{1}$.

${ }^{18} \mathrm{~A}$ large $\bar{\varepsilon}$ means that the $\varepsilon_{i}$ s are distributed over a wide range $[0, \bar{\varepsilon}]$. Note that $\bar{\varepsilon}=1 / g(0)$. For the interpretation of $1 / g(0)$ as a measure of luck in the symmetric tournament equilibrium (here: in state $s_{1}$ ) see Lazear (1995), p. 29.
} 
rational for each player to exert only minimal effort, because in this situation effort is not decisive for the tournament outcome but generates costs $c\left(e_{i}\right)$. Secondly, the parameter condition means that the cost parameter $k$ is large. This would strengthen the players' incentives to exert only little effort. At last, the left side of the parameter condition shows that the benefit $B$ has to be small. This would also result in minimal work incentives, because the winner prize $B$ is the driving force for exerting effort in tournaments. Altogether, a large $\bar{\varepsilon}$, a large $k$, and a small $B$ imply that it is rational to withhold effort, because winning the tournament is not very attractive and effort does not have any real influence on the outcome of the tournament but generates considerable disutility of effort. Therefore, it can be profitable to form a team, which leads to free riding and diseconomies of scope, because both effects increase the incentives to exert minimal effort. In such a situation, $I_{1}$ and $E$ bind themselves to reduce effort and the disutility of effort by forming a team. ${ }^{19}$ In other words, $I_{1}$ and $E$ form a team not in spite of but because of the free-rider effect and diseconomies of scope. The result of Proposition 1(iii) can be explained in an analogous way. Here, we also have a large $\bar{\varepsilon}$, a large $k$, and a small $B$ in the given parameter condition. In analogy, it is rational for the players to choose the organizational form which minimizes their efforts and thereby their disutilities of effort. But now, the formation of a team would lead to high efforts due to the cost effect (i.e. $\gamma_{I_{1}}, \gamma_{I_{2}}>1$ ). Therefore, the optimal strategy is not to form a team. Interestingly, the parameter condition of Proposition 1(iii) is stronger than the condition of Proposition 1(ii), as $27 B^{2}+3 B k \bar{\varepsilon}^{2}<2 k^{2} \bar{\varepsilon}^{4} \Longleftrightarrow \frac{27 B^{2}}{k \bar{\varepsilon}^{2}}+3 B<2 k \bar{\varepsilon}^{2}$. That

\footnotetext{
${ }^{19}$ From Section 2 we know that the effort of $I_{1}$ (and $E$ ) is $\frac{B}{k \bar{\varepsilon}}$ when not forming a team (state $s_{1}$ ) and $\frac{\gamma_{I_{1}}}{2} \frac{B \bar{\varepsilon}}{B-B \gamma_{I_{1}}+k \bar{\varepsilon}^{2}}$ when forming a team (state $s_{3}$ ). As long as $\gamma_{I_{1}}$ is small enough, i.e. the diseconomies of scope are large enough, there will be less effort if $I_{1}$ and $E$ form a team.
} 
means a mismatch III equilibrium is less likely (i.e., it holds for a smaller range of parameter values) than a mismatch II equilibrium. Considering the arguments above this result seems to be plausible, because formation of a team already leads to lower efforts according to the free-rider effect. Thus, in this context the "benefits of free riding" have to be compensated before switching to self-employment. This makes the existence of a mismatch III equilibrium less likely.

For simplicity, the analysis has been restricted to team contracts in form of equal sharing arrangements. In this setting, the case of Rickey Henderson illustrated in the introduction cannot be explained, because a mismatch I equilibrium does not exist. However, the Rickey Henderson case can be derived by allowing the possibility of entrance fees, which can be charged by $I_{1}, I_{2}$, or $E$ when forming a team:

\section{Proposition 2}

If there is symmetric information but entrance fees are allowed in team contracts, a mismatch I equilibrium will become possible.

\section{Proof. See Appendix B.}

The proof of Proposition 2 shows that a mismatch I equilibrium will become possible for purely strategic reasons if $I_{1}$ is allowed to offer an entrance fee in addition to the equal sharing agreement. Then, for a sufficiently high entrance fee $E$ will accept $I_{1}$ 's offer in spite of larger efficiency gains from a team contract with $I_{2}$ (i.e. $\gamma_{I_{2}}>\gamma_{I_{1}}$ ). The crucial point in this sort of strategic mismatching is the fact that $I_{2}$ has no chance to prevent $E$ from accepting $I_{1}$ 's offer despite of $\gamma_{I_{2}}>\gamma_{I_{1}}$. Player $I_{2}$ could promise to add an entrance fee to his equal sharing offer, too, but such a promise could not be credible. At $t_{3}$ of the sequential game, $I_{2}$ would never offer an entrance 
fee in addition to the standard equal sharing contract, because at this point of time $E$ cannot threat $I_{2}$ to form a team with $I_{1}$. Player $E$ anticipates this in $t_{2}$ when he decides about accepting or rejecting $I_{1}$ 's offer.

The proof of Proposition 2 also points out, which parameter constellations make a mismatch I equilibrium (with entrance fee) more likely. For example, $\gamma_{I_{2}}$ has to be high enough for a mismatch I. A high $\gamma_{I_{2}}$ means that offering a team contract to $E$ would be profitable for $I_{2}$. In addition, a high $\gamma_{I_{2}}$ results in large competitive disadvantages for $I_{1}$ when $I_{2}$ and $E$ form a team in this situation. This effect is indicated by equation (22), which shows that $E U_{I_{1}}^{+}\left(e_{I_{1}}^{*} ; s_{2}\right)$ is decreasing in $\gamma_{I_{2}}$. Therefore, if $\gamma_{I_{2}}$ is sufficiently high player $I_{1}$ will have strong incentives to prevent a team $\left\{I_{2}, E\right\}$. This is just the Rickey Henderson case considered in the introduction. Furthermore, the proof of Proposition 2 shows that a mismatch I equilibrium becomes more likely for a high $\gamma_{I_{1}}$ too. A high $\gamma_{I_{1}}$ guarantees that there are also considerable efficiency gains for $I_{1}$ and $E$ when forming a team. This makes $E$ more likely to accept $I_{1}$ 's equal sharing offer and, in addition, allows $I_{1}$ to pay an entrance fee that is sufficiently high for $E$ to forego an efficient match with $I_{2}$.

To sum up, the Propositions 1 and 2 have demonstrated that the three types of mismatches are possible for strategic reasons in sequential team contracting, although all players are completely informed. The next two subsections add asymmetric information to the analysis for discussing the question whether the perils of mismatching do increase or not under additional information problems.

\subsection{Uninformed Incumbents}

In this subsection, the assumption of completely informed players is revised. Now, we assume that the entrant $E$ still has complete information whereas 
the incumbents $I_{1}$ and $I_{2}$ are only provided with estimates of $\gamma_{I_{1}}$ and $\gamma_{I_{2}}$. These estimates are denoted by $\hat{\gamma}_{I_{1}}$ and $\hat{\gamma}_{I_{2}}$, respectively. We can imagine that nearly any mismatch problem can be generated by introducing sufficient uncertainty and asymmetric information. Thus, the asymmetric information problem will be restricted to the case where $I_{1}$ and $I_{2}$ have unbiased estimates of $\gamma_{I_{1}}$ and $\gamma_{I_{2}}$. In other words, $E$ knows the true values $\gamma_{I_{1}}$ and $\gamma_{I_{2}}$ for sure, whereas both $I_{1}$ and $I_{2}$ have the same estimates $\hat{\gamma}_{I_{1}}$ and $\hat{\gamma}_{I_{2}}$ that are statistically unbiased in the sense of $E\left[\hat{\gamma}_{I_{1}} \mid \gamma_{I_{1}}\right]=\gamma_{I_{1}}$ and $E\left[\hat{\gamma}_{I_{2}} \mid \gamma_{I_{2}}\right]=\gamma_{I_{2}}{ }^{20}$ In addition, for simplicity it is assumed that all uncertainty is resolved in $t_{5}$ before the tournament starts. Hence, we can still use the preliminary results of Section 2. This assumption is quite strong, but it helps to concentrate on the problem of mismatching. Mismatches can arise during $t_{1}-t_{4}$, before the tournament starts. Therefore, we assume asymmetric information only for this part of the sequential game. Furthermore, as information problems will play a dominant role all types of mismatches can be modelled by using suitable probability distributions and information structures. In this context, we obtain the following results:

\section{Proposition 3}

If the entrant $E$ is completely informed and the incumbents $I_{1}$ and $I_{2}$ only have unbiased estimates of $\gamma_{I_{1}}$ and $\gamma_{I_{2}}$ before $t_{5}$, the following will hold:

(i) There does not exist a mismatch I equilibrium.

(ii) There is the possibility of a mismatch II equilibrium. If $E U_{I_{1}}^{-}\left(e_{I_{1}}^{*} ; s_{3}\right)$ is concave in $\gamma_{I_{1}}$ and $E U_{I_{2}}^{-}\left(e_{I_{2}}^{*} ; s_{2}\right)$ is convex in $\gamma_{I_{2}}$ a mismatch II equilibrium will become less likely compared to the situation with

\footnotetext{
${ }^{20}$ Here, $E[\cdot]$ denotes the expectation operator.
} 
symmetric information. If $E U_{I_{2}}^{-}\left(e_{I_{2}}^{*} ; s_{2}\right)$ is concave in $\gamma_{I_{2}}$ a mismatch II equilibrium will become more likely.

(iii) There is the possibility of a mismatch III equilibrium. A mismatch III is more likely compared to the situation with symmetric information.

\section{Proof. See Appendix B.}

Comparing Proposition 3 and Proposition 1 we see that introducing asymmetric information leads to mixed results. On the one hand, a mismatch I equilibrium does not exist with uninformed incumbents as well as with symmetrically distributed information. In both situations, the entrant $E$ is able to verify that he will gain from forming a team with $I_{2}$. Hence, he will always reject $I_{1}$ 's offer in $t_{2}$.

On the other hand, it is not clear whether a mismatch II equilibrium is more likely under symmetric or under asymmetric information. The proof of Proposition 3(ii) shows that in spite of unbiased estimates $\hat{\gamma}_{I_{1}}$ and $\hat{\gamma}_{I_{2}}$ there are two decision anomalies which influence the matching process during $t_{1}-t_{4}$. The first anomaly is the well-known winner's curse which follows from the convexity of $E U_{I_{2}}^{-}\left(e_{I_{2}}^{*} ; s_{2}\right)$ and $E U_{I_{1}}^{-}\left(e_{I_{1}}^{*} ; s_{3}\right)$ concerning $\gamma_{I_{2}}$ or $\gamma_{I_{1}}$, respectively, by applying Jensen's Inequality. ${ }^{21}$ Here, a winner's curse means that $I_{2}$ and $I_{1}$ overestimate the gains from team formation (in spite of unbiased estimates), as $E_{\hat{\gamma}_{I_{2}}}\left[E U_{I_{2}}^{-}\left(e_{I_{2}}^{*} ; s_{2}\right)\right]>E U_{I_{2}}^{-}\left(e_{I_{2}}^{*} ; s_{2}\right)$ and $E_{\hat{\gamma}_{I_{1}}}\left[E U_{I_{1}}^{-}\left(e_{I_{1}}^{*} ; s_{3}\right)\right]>E U_{I_{1}}^{-}\left(e_{I_{1}}^{*} ; s_{3}\right)$. Interestingly, the winner's curse lessens

\footnotetext{
${ }^{21}$ The winner's curse is known from the bidding literature; see, e.g., Milgrom (1981). Consider, for example, a common value auction between $n$ bidders. Each bidder $i$ $(i=1, \ldots, n)$ is assumed to have an unbiased estimate $x_{i}$ of the unknown true value $v$ of the object to be auctioned, i.e. $E\left[x_{i} \mid v\right]=v$. Then the estimate of the winning bidder, $\max x_{i}$, is too optimistic as $E\left[\max x_{i} \mid v\right]>\max E\left[x_{i} \mid v\right]=v$ because of the convexity of the "max"-function and Jensen's Inequality; see Milgrom (1985), p. 265.
} 
the probability of a mismatch II, because now player $I_{2}$ is too optimistic when he decides about offering a team contract to $E$ (see inequality (A31)). But this effect will result in a more severe mismatching, because $\gamma_{I_{2}}<\gamma_{I_{1}}$ $<1$ indicates that a team $\left\{I_{2}, E\right\}$ is "more inefficient" than a team $\left\{I_{1}, E\right\}$. The winner's curse concerning player $I_{1}$ has no influence as condition (A33) (for $E$ accepting $I_{1}$ 's offer) is stronger than inequality (A32). Altogether, the winner's curse results in a lower probability for a strategic mismatch II at the expense of a higher probability for mismatching due to informational reasons. The second decision anomaly can be characterized as loser's curse. ${ }^{22}$ A loser's curse will arise if $E U_{I_{2}}^{-}\left(e_{I_{2}}^{*} ; s_{2}\right)$ and $E U_{I_{1}}^{-}\left(e_{I_{1}}^{*} ; s_{3}\right)$ are concave in $\gamma_{I_{2}}$ or $\gamma_{I_{1}}$, respectively. This results in $I_{1}$ and $I_{2}$ underestimating the gains from team formation. The effect of the loser's curse is ambivalent, because the loser's curse concerning $I_{1}$ makes a mismatch II less likely whereas a loser's curse concerning $I_{2}$ increases the perils of mismatching.

At last, according to Proposition 3(iii) the probability of a mismatch III equilibrium has increased compared to Proposition 1(iii). As the proof of Proposition 3(iii) shows, this result follows from $I_{1}$ and $I_{2}$ both being affected by a loser's curse when calculating the gains from team formation. Now, both players underestimate the benefits of team work, which reinforces the arguments given in the discussion following Proposition 1. Here, strategic mismatching works in the same direction as mismatching due to asymmetric information.

\subsection{Uninformed Entrant}

This scenario considers the case where the incumbents $I_{1}$ and $I_{2}$ are completely informed whereas $E$ only has two unbiased estimates $\hat{\gamma}_{I_{1}}$ and

\footnotetext{
${ }^{22}$ For the loser's curse see Holt and Sherman (1994).
} 
$\hat{\gamma}_{I_{2}}$ with $E\left[\hat{\gamma}_{I_{1}} \mid \gamma_{I_{1}}\right]=\gamma_{I_{1}}$ and $E\left[\hat{\gamma}_{I_{2}} \mid \gamma_{I_{2}}\right]=\gamma_{I_{2}}$. Again, we assume that asymmetric information is completely resolved before the tournament starts in $t_{5}$. Now, we obtain the following results:

\section{Proposition 4}

If the incumbents $I_{1}$ and $I_{2}$ are completely informed and the entrant $E$ only has unbiased estimates of $\gamma_{I_{1}}$ and $\gamma_{I_{2}}$ before $t_{5}$, the following will hold:

(i) There is the possibility of a mismatch I equilibrium.

(ii) There is the possibility of a mismatch II equilibrium. A mismatch II is less likely compared to the situation with symmetric information.

(iii) There is the possibility of a mismatch III equilibrium. A mismatch III is as likely as in the situation with symmetric information.

\section{Proof. See Apendix B.}

Proposition 4(i) supports the initial claim from the beginning of Subjection 3.2 that nearly any mismatch problem can be generated by assuming sufficient uncertainty and asymmetric information. Propositions 1 and 3 have shown that, as long as entrance fees are not allowed, a mismatch I can never exist for strategic reasons, because the entrant $E$ will always choose the efficient match with $I_{2}$ to maximize expected utility. According to the proof of Proposition 4(i), with an uninformed entrant a mismatch I will become possible as long as the "relative loser's curse" $\Delta_{I_{2}}-\Delta_{I_{1}}$ is large enough. But such a mismatch I would solely be due to information problems. Proposition 4(ii) contains the interesting result that assuming an uninformed entrant $E$ makes a mismatch II less likely compared to the symmetric information case. Here, the information problem of player $E$ can cause a loser's curse that works against strategic mismatching, because $E$ 
underestimates the potential gains of a (strategic) mismatch II. This result points out that a decision anomaly like the loser's curse may be welfare improving in some situations. The result of Proposition 4(iii) is exactly the same as the result of Proposition 1(iii), because in both cases no decision node of player $E$ is reached in the game. Therefore, it does not matter whether $E$ has complete information or not.

To sum up, the welfare effects of introducing an uninformed entrant $E$ are not clear. On the one hand, this kind of asymmetric information makes a mismatch I possible, which cannot occur when $E$ has complete information and entrance fees are not allowed (see Proposition 1(i)). On the other hand, a mismatch II equilibrium becomes less likely compared to the symmetric information case of Proposition 1(ii).

\section{Conclusions}

The results of this paper show that mismatches are not solely caused by information problems but are also due to strategic reasons. Mismatching will be rational, if the costs from mismatching are dominated by the benefits of mismatching based on strategic considerations. For example, forming an inefficient team can be beneficial when the resulting disadvantages for competing teams are larger than the own efficiency loss. Another result of this paper shows that voluntary mismatching can be rational to minimize work incentives when effort only plays a subordinate role in tournament competition (caused by the dominant influence of luck) but the marginal costs of effort are high.

This paper also points in two further directions. First, comparing the results of this paper and the results derived in Kräkel (1999), it becomes 
clear that the kind of competition at the final stage of the game can play a major role in strategic mismatching. Kräkel (1999) shows that a mismatch II equilibrium (i.e., forming an inefficient team) cannot be possible - neither assuming symmetric nor asymmetric information - when considering Cournot competition at the final stage. This result seems to be intuitively plausible because both the free-rider effect and diseconomies of scope work in the same direction and make a mismatch II unprofitable. In this paper, tournament competition takes place at the final stage. However, this kind of competition makes a mismatch II equilibrium possible, especially because of its luck component. ${ }^{23}$ Secondly, this paper combines strategic mismatching with the problem of decision anomalies in form of the winner's curse and the loser's curse, respectively. Both kinds of bidding failures are originally known from the auction literature, but also arise in other economic contexts when there is bidding under asymmetric information (e.g., bidding in takeover contests, see Roll (1986), or bidding for workers in the labor market, see Milgrom and Oster (1987)). In the model considered here there is also a sort of bidding under asymmetric information when the incumbents bid for the new entrant by offering a team contract. The results show that overestimating (winner's curse) and underestimating (loser's curse) the potential gains from team formation may be welfare improving or not depending on whether the decision anomalies mitigate or reinforce the incentives for strategic mismatching.

\footnotetext{
${ }^{23} \mathrm{~A}$ tournament can be interpreted as a kind of lottery where only the winner receives a high prize. The outcome of this lottery can be influenced by the competitors' efforts. Therefore, tournaments are an extreme form of competition.
} 


\section{Appendix}

Appendix A: Derivation of $g(x), h(y)$, and $H(y)$

The density $g(\cdot)$ of the random variable $X=\varepsilon_{(2)}-\varepsilon_{i}$ is the convolution of the densities for $\varepsilon_{(2)}$ and $\varepsilon_{i}$, where $f\left(\varepsilon_{i}\right)=1 / \bar{\varepsilon}$ by assumption. The density of the highest of two order statistics is $f_{(2)}\left(\varepsilon_{(2)}\right)=2 F\left(\varepsilon_{(2)}\right) f\left(\varepsilon_{(2)}\right)=$ $2 \varepsilon_{(2)} / \bar{\varepsilon}^{2} \cdot{ }^{24}$ Because $\varepsilon_{(2)}$ and $\varepsilon_{i}$ are stochastically independent, we obtain $g(x)=\int f_{(2)}\left(\varepsilon_{(2)}\right) f\left(\varepsilon_{(2)}-x\right) d \varepsilon_{(2)}=\int 2 \varepsilon_{(2)} / \bar{\varepsilon}^{3} d \varepsilon_{(2)}$. To determine the exact density function we need the concrete limits of the integral. We know that $0 \leq \varepsilon_{(2)} \leq \bar{\varepsilon}$ and $0 \leq \varepsilon_{i} \leq \bar{\varepsilon} \Longleftrightarrow 0 \leq \varepsilon_{(2)}-x \leq \bar{\varepsilon} \Longleftrightarrow x \leq \varepsilon_{(2)} \leq \bar{\varepsilon}+x$. The random variable $X=\varepsilon_{(2)}-\varepsilon_{i}$ is distributed over the interval $[-\bar{\varepsilon}, \bar{\varepsilon}]$, because each of the random variables $\varepsilon_{(2)}$ and $\varepsilon_{i}$ can be 0 or $\bar{\varepsilon}$ in the worst and in the best case, respectively. The interval $[-\bar{\varepsilon}, \bar{\varepsilon}]$ can be divided into the two subintervals $-\bar{\varepsilon} \leq x \leq 0$ and $0<x \leq \bar{\varepsilon}$. Combining these two subintervals with the two conditions $0 \leq \varepsilon_{(2)} \leq \bar{\varepsilon}$ and $x \leq \varepsilon_{(2)} \leq \bar{\varepsilon}+x$, which must hold at the same time, gives $0 \leq \varepsilon_{(2)} \leq \bar{\varepsilon}+x$ for the first subinterval and $x \leq \varepsilon_{(2)} \leq \bar{\varepsilon}$ for the second subinterval. Thus, we have

$$
\begin{aligned}
& g(x)=\left\{\begin{array}{lll}
\int_{0}^{\bar{\varepsilon}+x} \frac{2 \varepsilon_{(2)}}{\bar{\varepsilon}^{3}} d \varepsilon_{(2)} & \text { if } & -\bar{\varepsilon} \leq x \leq 0 \\
\int_{x}^{\bar{\varepsilon}} \frac{2 \varepsilon_{(2)}}{\bar{\varepsilon}^{3}} d \varepsilon_{(2)} & \text { if } & 0<x \leq \bar{\varepsilon}
\end{array}\right. \\
& =\left\{\begin{array}{cl}
\frac{(\bar{\varepsilon}+x)^{2}}{\bar{\varepsilon}^{3}} & \text { if } \quad-\bar{\varepsilon} \leq x \leq 0 \\
\frac{1}{\bar{\varepsilon}}-\frac{x^{2}}{\bar{\varepsilon}^{3}} & \text { if } \quad 0<x \leq \bar{\varepsilon} .
\end{array}\right.
\end{aligned}
$$

The density function $h(y)$ can be derived in analogy to $g(x)$. We obtain the following triangular density:

$$
h(y)=\left\{\begin{array}{lll}
\frac{\bar{\varepsilon}+y}{\bar{\varepsilon}^{2}} & \text { if } \quad-\bar{\varepsilon} \leq y \leq 0 \\
\frac{\bar{\varepsilon}-y}{\bar{\varepsilon}^{2}} & \text { if } \quad 0<y \leq \bar{\varepsilon}
\end{array}\right.
$$

\footnotetext{
${ }^{24}$ For the distribution of order statistics and functions of random variables see, e.g., Mood, Graybill and Boes (1974).
} 
Integrating $h(y)$ and noting that $H(-\bar{\varepsilon})=0, H(0)=\frac{1}{2}$, and $H(\bar{\varepsilon})=1$ yields

$$
H(y)=\left\{\begin{array}{lll}
\frac{y}{\bar{\varepsilon}}+\frac{y^{2}}{2 \bar{\varepsilon}^{2}}+\frac{1}{2} & \text { if } & -\bar{\varepsilon} \leq y \leq 0 \\
\frac{y}{\bar{\varepsilon}}-\frac{y^{2}}{2 \bar{\varepsilon}^{2}}+\frac{1}{2} & \text { if } & 0<y \leq \bar{\varepsilon}
\end{array}\right.
$$

\section{Appendix B: Proofs of Propositions 1-4}

Proof of Proposition 1:

Result (i) can be proved as follows. For a mismatch I equilibrium four conditions must hold:

$$
\begin{gathered}
E U_{I_{2}}^{+}\left(e_{I_{2}}^{*} ; s_{2}\right)=E U_{E}^{+}\left(e_{E}^{*} ; s_{2}\right)>E U_{i}\left(e_{i}^{*} ; s_{1}\right), \quad i \in\left\{I_{1}, I_{2}, E\right\} \\
E U_{I_{1}}^{+}\left(e_{I_{1}}^{*} ; s_{3}\right)>E U_{I_{1}}^{+}\left(e_{I_{1}}^{*} ; s_{2}\right) \\
E U_{E}^{+}\left(e_{E}^{*} ; s_{3}\right) \geq E U_{E}^{+}\left(e_{E}^{*} ; s_{2}\right) \\
\gamma_{I_{2}}>\gamma_{I_{1}}>1 .
\end{gathered}
$$

Inequality (A1) means that $I_{2}$ and $E$ prefer competing as a team $\left\{I_{2}, E\right\}$ in the tournament to competing as single players. Therefore, without the interference of player $I_{1}$ efficient matching would arise. Condition (A2) ensures that $I_{1}$ offers a contract to $E$ in $t_{1}$. Inequality (A3) guarantees that $E$ will accept this offer, because his expected utility is at least as great as in state $s_{2}$ when forming a team with $I_{2}$. Condition (A4) defines a mismatch I, where the team $\left\{I_{2}, E\right\}$ generates larger economies of scope than the team $\left\{I_{1}, E\right\}$. Result (i) holds, because (A3) and (A4) cannot be true at the same time: Equations (21) and (25) show that $E U_{E}^{+}\left(e_{E}^{*} ; s_{3}\right)$ and $E U_{E}^{+}\left(e_{E}^{*} ; s_{2}\right)$ are identical functions of $\gamma_{I_{1}}$ and $\gamma_{I_{2}}$, respectively. These functions are increasing in $\gamma_{I_{1}}$ and $\gamma_{I_{2}}$, respectively, so that (A3) and (A4) lead to a contradiction. 
Now, result (ii) is considered. There are three conditions for a mismatch II:

$$
\begin{gathered}
E U_{I_{2}}^{-}\left(e_{I_{2}}^{*} ; s_{2}\right)<E U_{i}\left(e_{i}^{*} ; s_{1}\right), \quad i \in\left\{I_{1}, I_{2}, E\right\} \\
E U_{I_{1}}^{-}\left(e_{I_{1}}^{*} ; s_{3}\right)=E U_{E}^{-}\left(e_{E}^{*} ; s_{3}\right)>E U_{i}\left(e_{i}^{*} ; s_{1}\right), \quad i \in\left\{I_{1}, I_{2}, E\right\} \\
\gamma_{I_{2}}<\gamma_{I_{1}}<1 .
\end{gathered}
$$

Inequality (A5) guarantees that $I_{2}$ does not want to form a team $\left\{I_{2}, E\right\}$. On the other hand, (A6) ensures that forming a team $\left\{I_{1}, E\right\}$ is rational for $I_{1}$ and $E$ compared to state $s_{1}$, where all players compete alone against each other in the tournament. At last, (A7) defines mismatch II. Substituting the concrete expressions for the expected utilities from (4), (17), and (23) into (A5) and (A6) we see that the conditions (A5) - (A7) are equivalent to

$$
\Psi\left(\gamma_{I_{2}}\right)<0, \quad \Psi\left(\gamma_{I_{1}}\right)>0, \quad \gamma_{I_{2}}<\gamma_{I_{1}}<1
$$

with

$$
\Psi(\gamma):=\frac{\Lambda(\gamma)}{\left(B-B \gamma+k \bar{\varepsilon}^{2}\right)^{2} k \frac{\bar{\varepsilon}^{2}}{B}}
$$

and

$$
\begin{aligned}
\Lambda(\gamma): & =\gamma^{2}\left(\frac{1}{2} B^{3}-\frac{1}{3} k \bar{\varepsilon}^{2} B^{2}\right)+\gamma\left(\frac{13}{24} k^{2} \bar{\varepsilon}^{4} B-\frac{1}{3} k \bar{\varepsilon}^{2} B^{2}-B^{3}\right) \\
& -\frac{1}{12} k^{3} \bar{\varepsilon}^{6}+\frac{2}{3} k \bar{\varepsilon}^{2} B^{2}-\frac{1}{6} k^{2} \bar{\varepsilon}^{4} B+\frac{1}{2} B^{3} .
\end{aligned}
$$

Note that the denominator of $\Psi(\gamma)$ in (A9) is positive so that (A8) is equivalent to

$$
\Lambda\left(\gamma_{I_{2}}\right)<0, \quad \Lambda\left(\gamma_{I_{1}}\right)>0, \quad \gamma_{I_{2}}<\gamma_{I_{1}}<1
$$


The function $\Lambda(\gamma)$ describes a convex or a concave parabola, respectively, depending on the sign of $\frac{1}{2} B^{3}-\frac{1}{3} k \bar{\varepsilon}^{2} B^{2}$. If this expression is positive (negative) the parabola will be convex (concave). Thus, there are two possibilities for (A11) to hold, which are sketched in Figure 2.

\section{[Figure 2]}

In any case, the parabola $\Lambda(\gamma)$ has the following two roots:

$$
\begin{aligned}
& \bar{\gamma}=\frac{-24 B^{2}+13 k^{2} \bar{\varepsilon}^{4}-8 B k \bar{\varepsilon}^{2}+\sqrt{144 B^{2} k^{2} \bar{\varepsilon}^{4}+105 k^{4} \bar{\varepsilon}^{8}-240 k^{3} \bar{\varepsilon}^{6} B}}{-2\left(12 B-8 k \bar{\varepsilon}^{2}\right) B} \\
& \overline{\bar{\gamma}}=\frac{-24 B^{2}+13 k^{2} \bar{\varepsilon}^{4}-8 B k \bar{\varepsilon}^{2}-\sqrt{144 B^{2} k^{2} \bar{\varepsilon}^{4}+105 k^{4} \bar{\varepsilon}^{8}-240 k^{3} \bar{\varepsilon}^{6} B}}{-2\left(12 B-8 k \bar{\varepsilon}^{2}\right) B} .
\end{aligned}
$$

Figure 2(a) shows the case where (A11) is fullfilled when the parabola $\Lambda(\gamma)$ is convex. There are two conditions that have to be met. The first condition guarantees convexity of of $\Lambda(\gamma)$ :

$$
\frac{1}{2} B^{3}-\frac{1}{3} k \bar{\varepsilon}^{2} B^{2}=\frac{B^{2}}{24}\left(12 B-8 k \bar{\varepsilon}^{2}\right)>0 .
$$

The second condition requires that the right-hand root of $\Lambda(\gamma)$ lies between 0 and 1 (see Figure 2(a)). Comparing (A12) and (A13) we see that $\overline{\bar{\gamma}}$ is the right-hand root: The denominator of $\bar{\gamma}$ or $\overline{\bar{\gamma}}$, respectively, is negative because of the convexity of $\Lambda(\gamma)$ (see (A14)). Therefore, for the right-hand root to lie between 0 and 1 either both the numerator of $\bar{\gamma}$ and the numerator of $\overline{\bar{\gamma}}$ have to be negative or one numerator has to be negative and the other one has to be positive. ${ }^{25}$ In both cases $\overline{\bar{\gamma}}$ is the right-hand (or larger) root because of

\footnotetext{
${ }^{25}$ In the last case, $\overline{\bar{\gamma}}$ is the root with the negative numerator whereas $\bar{\gamma}$ has a positive numerator. This follows from the sign in front of the square root in the numerators of (A12) and (A13).
} 
the negative denominator. This yields the following second condition:

$$
0<\overline{\bar{\gamma}}<1
$$

For $\overline{\bar{\gamma}}<1$ to be true the inequality

$$
\begin{aligned}
-24 B^{2}+13 k^{2} \bar{\varepsilon}^{4}-8 B k \bar{\varepsilon}^{2}-\sqrt{144 B^{2} k^{2} \bar{\varepsilon}^{4}+105 k^{4} \bar{\varepsilon}^{8}-240 k^{3} \bar{\varepsilon}^{6} B} & > \\
-2\left(12 B-8 k \bar{\varepsilon}^{2}\right) B & \Longleftrightarrow \\
-2 k \bar{\varepsilon}^{2}\left(12 B-\frac{13}{2} k \bar{\varepsilon}^{2}\right)-\sqrt{144 B^{2} k^{2} \bar{\varepsilon}^{4}+105 k^{4} \bar{\varepsilon}^{8}-240 k^{3} \bar{\varepsilon}^{6} B} & >0
\end{aligned}
$$

must hold. But this cannot be true, because both the first term (because of (A14)) and the second term on the left side of (A16) are negative.

Figure 2(b) shows the situation where (A11) is fulfilled when the parabola $\Lambda(\gamma)$ is concave. Again, we have two conditions that have to be met:

$$
\frac{1}{2} B^{3}-\frac{1}{3} k \bar{\varepsilon}^{2} B^{2}=\frac{B^{2}}{24}\left(12 B-8 k \bar{\varepsilon}^{2}\right)<0 \Longleftrightarrow 3 B<2 k \bar{\varepsilon}^{2},
$$

which guarantees concavity, and

$$
0<\overline{\bar{\gamma}}<1
$$

which means that left-hand root of $\Lambda(\gamma)$ lies between 0 and 1 (see Figure 2 (b)). ${ }^{26}$ (A18) can be rewritten as

$$
\begin{aligned}
-24 B k \bar{\varepsilon}^{2}+13 k^{2} \bar{\varepsilon}^{4} & <\sqrt{144 B^{2} k^{2} \bar{\varepsilon}^{4}+105 k^{4} \bar{\varepsilon}^{8}-240 k^{3} \bar{\varepsilon}^{6} B} \\
& <-24 B^{2}+13 k^{2} \bar{\varepsilon}^{4}-8 B k \bar{\varepsilon}^{2} \Rightarrow \\
3 B & <2 k \bar{\varepsilon}^{2}
\end{aligned}
$$

\footnotetext{
${ }^{26}$ Note that because of (A17) both the denominator of $\bar{\gamma}$ and the denominator of $\overline{\bar{\gamma}}$ are positive. Therefore, both numerators have to be positive, too, for condition (A11) to be met. $\overline{\bar{\gamma}}$ is the left-hand (or smaller) root of $\Lambda(\gamma)$ because of the sign of the square root in the numerators of (A12) and (A13).
} 
which will hold if (A17) is true. Altogether, if $\Lambda(\gamma)$ is convex, (A11) cannot be met. On the other hand, concavity of $\Lambda(\gamma)$ - i.e. (A17) holds - ensures that the left-hand root of $\Lambda(\gamma)$ lies between 0 and 1 so that mismatch II equilibria become possible.

At last, result (iii) has to be proved. This result requires three conditions to be met:

$$
\begin{gathered}
E U_{I_{2}}^{+}\left(e_{I_{2}}^{*} ; s_{2}\right)<E U_{i}\left(e_{i}^{*} ; s_{1}\right), \quad i \in\left\{I_{1}, I_{2}, E\right\} \\
E U_{I_{1}}^{+}\left(e_{I_{1}}^{*} ; s_{3}\right)<E U_{i}\left(e_{i}^{*} ; s_{1}\right), \quad i \in\left\{I_{1}, I_{2}, E\right\} \\
\gamma_{I_{1}}, \gamma_{I_{2}}>1 .
\end{gathered}
$$

The inequalities (A20) and (A21) mean that neither $I_{1}$ nor $I_{2}$ is interested in forming a team with $E$. Together with condition (A22) we have a mismatch III equilibrium, where $I_{1}$ and $E$ forego to form a profitable team. Using the concrete expressions for the expected utilities from (4), (21), and (25) we see that the conditions (A20)-(A22) are equivalent to

$$
\ni \gamma>1: \Omega(\gamma)<0
$$

where

$$
\begin{aligned}
\Omega(\gamma): & =\gamma^{2}\left(B^{3}+\frac{1}{3} k \bar{\varepsilon}^{2} B^{2}\right)+\gamma\left(\frac{4}{3} k \bar{\varepsilon}^{2} B^{2}+\frac{5}{12} k^{2} \bar{\varepsilon}^{4} B-2 B^{3}\right) \\
& +\frac{1}{3} k^{2} \bar{\varepsilon}^{4} B-\frac{5}{3} k \bar{\varepsilon}^{2} B^{2}-\frac{1}{6} k^{3} \bar{\varepsilon}^{6}+B^{3} .
\end{aligned}
$$

The convex parabola described by (A24) has two roots:

$$
\gamma^{\prime}=\frac{24 B^{2}-5 k^{2} \bar{\varepsilon}^{4}-16 B k \bar{\varepsilon}^{2}+\sqrt{57 k^{4} \bar{\varepsilon}^{8}+192 k^{3} \bar{\varepsilon}^{6} B+144 k^{2} \bar{\varepsilon}^{4} B^{2}}}{8 B\left(k \bar{\varepsilon}^{2}+3 B\right)}
$$




$$
\gamma^{\prime \prime}=\frac{24 B^{2}-5 k^{2} \bar{\varepsilon}^{4}-16 B k \bar{\varepsilon}^{2}-\sqrt{57 k^{4} \bar{\varepsilon}^{8}+192 k^{3} \bar{\varepsilon}^{6} B+144 k^{2} \bar{\varepsilon}^{4} B^{2}}}{8 B\left(k \bar{\varepsilon}^{2}+3 B\right)} .
$$

Therefore, the minimum of the parabola $\Omega(\gamma)$ lies below the horizontal axis. In this case, (A23) means that the right-hand root of $\Omega(\gamma)$ must be greater than 1, i.e. $\gamma^{\prime}>1$. This condition can be simplified to

$$
\begin{aligned}
\sqrt{57 k^{2} \bar{\varepsilon}^{4}+192 B k \bar{\varepsilon}^{2}+144 B^{2}} & >5 k \bar{\varepsilon}^{2}+24 B \Longleftrightarrow \\
27 B^{2}+3 B k \bar{\varepsilon}^{2} & <2 k^{2} \bar{\varepsilon}^{4} .
\end{aligned}
$$

Proof of Proposition 2:

Consider the case where $I_{1}$ offers $E$ an entrance fee $\eta>0$ in addition to the equal sharing arrangement to make him sign the team contract. Thereby the conditions (A1)-(A4) from the proof of Proposition 1 must be rewritten as

$$
\begin{gathered}
E U_{I_{2}}^{+}\left(e_{I_{2}}^{*} ; s_{2}\right)=E U_{E}^{+}\left(e_{E}^{*} ; s_{2}\right)>E U_{i}\left(e_{i}^{*} ; s_{1}\right), \quad i \in\left\{I_{1}, I_{2}, E\right\} \\
E U_{I_{1}}^{+}\left(e_{I_{1}}^{*} ; s_{3}\right)-\eta>E U_{I_{1}}^{+}\left(e_{I_{1}}^{*} ; s_{2}\right) \\
E U_{E}^{+}\left(e_{E}^{*} ; s_{3}\right)+\eta \geq E U_{E}^{+}\left(e_{E}^{*} ; s_{2}\right) \\
\gamma_{I_{2}}>\gamma_{I_{1}}>1 .
\end{gathered}
$$

In this situation, $I_{1}$ has all the bargaining power, because in $t_{1}$ he makes a take-it-or-leave-it offer to $E$, whereas $E$ has $E U_{E}^{+}\left(e_{E}^{*} ; s_{2}\right)$ as reservation value and can only choose between accepting or rejecting the offer in $t_{2}$. Therefore, $I_{1}$ chooses $\eta$ to make $E$ just indifferent between his offer and forming a team with $I_{2}$, i.e. $\eta=E U_{E}^{+}\left(e_{E}^{*} ; s_{2}\right)-E U_{E}^{+}\left(e_{E}^{*} ; s_{3}\right)$. After substituting for the 
expected utilities in (A25)-(A27) we obtain three conditions for a mismatch I equilibrium with entrance fee:

$$
\begin{gathered}
\frac{1}{8} B \frac{4 B^{2}\left(1-\gamma_{I_{2}}\right)^{2}+B k \bar{\varepsilon}^{2}\left(7 \gamma_{I_{2}}-8\right)+2 k^{2} \bar{\varepsilon}^{4}}{\left(B \gamma_{I_{2}}-B+k \bar{\varepsilon}^{2}\right)^{2}}>\frac{B}{3}-\frac{B^{2}}{2 k \bar{\varepsilon}^{2}} \\
E U_{I_{1}}^{+}\left(e_{I_{1}}^{*} ; s_{3}\right)-E U_{E}^{+}\left(e_{E}^{*} ; s_{2}\right)+E U_{E}^{+}\left(e_{E}^{*} ; s_{3}\right)>E U_{I_{1}}^{+}\left(e_{I_{1}}^{*} ; s_{2}\right) \Longleftrightarrow \\
2\left[4 B^{2}\left(1-\gamma_{I_{1}}\right)^{2}+B k \bar{\varepsilon}^{2}\left(7 \gamma_{I_{1}}-8\right)+2 k^{2} \bar{\varepsilon}^{4}\right]\left(B \gamma_{I_{2}}-B+k \bar{\varepsilon}^{2}\right)^{2} \\
-4 k \bar{\varepsilon}^{2}\left(k \bar{\varepsilon}^{2}-B\right)\left(B \gamma_{I_{1}}-B+k \bar{\varepsilon}^{2}\right)^{2} \\
-\left[4 B^{2}\left(1-\gamma_{I_{2}}\right)^{2}+B k \bar{\varepsilon}^{2}\left(7 \gamma_{I_{2}}-8\right)+2 k^{2} \bar{\varepsilon}^{4}\right]\left(B \gamma_{I_{1}}-B+k \bar{\varepsilon}^{2}\right)^{2} \\
>0 \\
0 \\
\gamma_{I_{2}}>\gamma_{I_{1}}>1 .
\end{gathered}
$$

These conditions hold for a lot of feasible parameter constellations which can easily be checked by a numerical example (let, e.g., $k=2, \bar{\varepsilon}=2, B=1$, $\left.\gamma_{I_{2}}=5, \gamma_{I_{1}}=4.5\right)$

\section{Proof of Proposition 3:}

The result of Propositon 3(i) can be proved in analogy to Proposition 1(i). Again, because of complete information the entrant $E$ is able to recognize in $t_{2}$ that he will gain from a match with $I_{2}$ compared to a match with $I_{1}$. Therefore, he will always reject $I_{1}$ 's offer in $t_{2}$ (without additional entrance fee).

The result (ii) is different from the corresponding result in Proposition 1. Here, a mismatch II equilibrium requires the following conditions to hold:

$$
E_{\hat{\gamma}_{I_{2}}}\left[E U_{I_{2}}^{-}\left(e_{I_{2}}^{*} ; s_{2}\right)\right]<E U_{i}\left(e_{i}^{*} ; s_{1}\right), \quad i \in\left\{I_{1}, I_{2}, E\right\}
$$




$$
\begin{gathered}
E_{\hat{\gamma}_{I_{1}}}\left[E U_{I_{1}}^{-}\left(e_{I_{1}}^{*} ; s_{3}\right)\right]>E U_{i}\left(e_{i}^{*} ; s_{1}\right), \quad i \in\left\{I_{1}, I_{2}, E\right\} \\
E U_{E}^{-}\left(e_{E}^{*} ; s_{3}\right)>E U_{i}\left(e_{i}^{*} ; s_{1}\right), \quad i \in\left\{I_{1}, I_{2}, E\right\} \\
\gamma_{I_{2}}<\gamma_{I_{1}}<1,
\end{gathered}
$$

where $E_{\circ}$ denotes the expectation operator with respect to "॰". Note that $E U_{I_{2}}^{-}\left(e_{I_{2}}^{*} ; s_{2}\right)$ and $E U_{I_{1}}^{-}\left(e_{I_{1}}^{*} ; s_{3}\right)$ are identical functions of $\gamma_{I_{2}}$ and $\gamma_{I_{1}}$, respectively:

$$
\Gamma(\gamma):=\frac{1}{8} B k \bar{\varepsilon}^{2} \frac{2 k \bar{\varepsilon}^{2}-B \gamma}{\left(B-B \gamma+k \bar{\varepsilon}^{2}\right)^{2}}
$$

with $\gamma=\gamma_{I_{1}}, \gamma_{I_{2}}$ and $\Gamma^{\prime \prime}(\gamma)=\frac{1}{4} B^{3} k \bar{\varepsilon}^{2}\left[-2 B-B \gamma+4 k \bar{\varepsilon}^{2}\right] /\left[B-B \gamma+k \bar{\varepsilon}^{2}\right]^{2}$. We see that $\Gamma^{\prime \prime}(\gamma)$ is positive for $\gamma<\frac{4 k \bar{\varepsilon}^{2}-2 B}{B}$ and negative for $\gamma>\frac{4 k \bar{\varepsilon}^{2}-2 B}{B}$. Therefore, $\Gamma(\gamma)$ is convex $\left(\Gamma^{\prime \prime}(\gamma)>0\right)$ or concave $\left(\Gamma^{\prime \prime}(\gamma)<0\right)$ depending on the magnitude of $\gamma$. Hence, applying Jensen's Inequality yields

$$
\begin{gathered}
E_{\hat{\gamma}_{I_{2}}}\left[E U_{I_{2}}^{-}\left(e_{I_{2}}^{*} ; s_{2}\right)\right] \gtrless E U_{I_{2}}^{-}\left(e_{I_{2}}^{*} ; s_{2}\right), \quad \text { if } \quad \gamma_{I_{2}} \lessgtr \frac{4 k \bar{\varepsilon}^{2}-2 B}{B} \\
E_{\hat{\gamma}_{I_{1}}}\left[E U_{I_{1}}^{-}\left(e_{I_{1}}^{*} ; s_{3}\right)\right] \gtrless E U_{I_{1}}^{-}\left(e_{I_{1}}^{*} ; s_{3}\right), \quad \text { if } \quad \gamma_{I_{1}} \lessgtr \frac{4 k \bar{\varepsilon}^{2}-2 B}{B} .
\end{gathered}
$$

Proposition 1(ii) has shown that a mismatch II becomes possible for certain parameter values when $\gamma_{I_{1}}$ and $\gamma_{I_{2}}$ are known for sure by $I_{1}$ and $I_{2}$. If $E U_{I_{1}}^{-}\left(e_{I_{1}}^{*} ; s_{3}\right)$ is concave in $\gamma_{I_{1}}$ and $E U_{I_{2}}^{-}\left(e_{I_{2}}^{*} ; s_{2}\right)$ is convex in $\gamma_{I_{2}}$ the inequalities (A31) and (A32) will be stronger than the inequalities (A5) and (A6) in the proof of Proposition 1(ii) (i.e., a mismatch II will become less likely). In this situation, (A33) is irrelevant, because $E U_{E}^{-}\left(e_{E}^{*} ; s_{3}\right)$ $\equiv E U_{I_{1}}^{-}\left(e_{I_{1}}^{*} ; s_{3}\right)$ and thereby (A32) implies (A33). On the other hand, if $E U_{I_{2}}^{-}\left(e_{I_{2}}^{*} ; s_{2}\right)$ is concave in $\gamma_{I_{2}}$ the condition (A5) will be stronger than (A31) 
(i.e., a mismatch II will become more likely). The case where $E U_{I_{1}}^{-}\left(e_{I_{1}}^{*} ; s_{3}\right)$ is convex in $\gamma_{I_{1}}$ is unimportant, because now (A33) implies (A32).

The result of Proposition 3(iii) follows from the fact that $E U_{I_{2}}^{+}\left(e_{I_{2}}^{*} ; s_{2}\right)$ and $E U_{I_{1}}^{+}\left(e_{I_{1}}^{*} ; s_{3}\right)$ are identical functions of $\gamma_{I_{2}}$ or $\gamma_{I_{1}}$ which are both concave in $\gamma_{I_{2}}$ or $\gamma_{I_{1}}$, respectively, because of $\partial^{2} E U_{I_{2}}^{+}\left(e_{I_{2}}^{*} ; s_{2}\right) / \partial \gamma_{I_{2}}^{2}<0$ and $\partial^{2} E U_{I_{1}}^{+}\left(e_{I_{1}}^{*} ; s_{3}\right) / \partial \gamma_{I_{1}}^{2}<0$. Applying Jensen's Inequality we obtain $E_{\hat{\gamma}_{I_{2}}}\left[E U_{I_{2}}^{+}\left(e_{I_{2}}^{*} ; s_{2}\right)\right]<E U_{I_{2}}^{+}\left(e_{I_{2}}^{*} ; s_{2}\right)$ and $E_{\hat{\gamma}_{I_{1}}}\left[E U_{I_{1}}^{+}\left(e_{I_{1}}^{*} ; s_{3}\right)\right]<E U_{I_{1}}^{+}\left(e_{I_{1}}^{*} ; s_{3}\right)$. Hence, the two inequalities (A20) and (A21) become more likely to hold, which proves Proposition 3(iii).

\section{Proof of Proposition 4:}

The result of Propositon 4(i) considers the case of a mismatch I equilibrium. In analogy to the proof of Proposition 1(i), the conditions of a mismatch I are:

$$
\begin{gathered}
E U_{I_{2}}^{+}\left(e_{I_{2}}^{*} ; s_{2}\right)>E U_{i}\left(e_{i}^{*} ; s_{1}\right), \quad i \in\left\{I_{1}, I_{2}, E\right\} \\
E_{\hat{\gamma}_{I_{2}}}\left[E U_{E}^{+}\left(e_{E}^{*} ; s_{2}\right)\right]>E U_{i}\left(e_{i}^{*} ; s_{1}\right), \quad i \in\left\{I_{1}, I_{2}, E\right\} \\
E U_{I_{1}}^{+}\left(e_{I_{1}}^{*} ; s_{3}\right)>E U_{I_{1}}^{+}\left(e_{I_{1}}^{*} ; s_{2}\right) \\
E_{\hat{\gamma}_{I_{1}}}\left[E U_{E}^{+}\left(e_{E}^{*} ; s_{3}\right)\right] \geq E_{\hat{\gamma}_{I_{2}}}\left[E U_{E}^{+}\left(e_{E}^{*} ; s_{2}\right)\right] \\
\gamma_{I_{2}}>\gamma_{I_{1}}>1 .
\end{gathered}
$$

Note that $E U_{I_{2}}^{+}\left(e_{I_{2}}^{*} ; s_{2}\right), E U_{E}^{+}\left(e_{E}^{*} ; s_{2}\right), E U_{I_{1}}^{+}\left(e_{I_{1}}^{*} ; s_{3}\right)$, and $E U_{E}^{+}\left(e_{E}^{*} ; s_{3}\right)$ are identical functions of $\gamma_{I_{2}}$ or $\gamma_{I_{1}}$ which are concave in $\gamma_{I_{2}}$ or $\gamma_{I_{1}}$, respectively (i.e., the second derivative with respect to $\gamma_{I_{2}}$ or $\gamma_{I_{1}}$ is negative). Therefore, 
by applying Jensen's Inequality and substituting for the expected utilities (A36)-(A40) can be written $\operatorname{as}^{27}$

$$
\begin{gathered}
\frac{1}{8} B \frac{4 B^{2}\left(1-\gamma_{I_{2}}\right)^{2}+B k \bar{\varepsilon}^{2}\left(7 \gamma_{I_{2}}-8\right)+2 k^{2} \bar{\varepsilon}^{4}}{\left(B \gamma_{I_{2}}-B+k \bar{\varepsilon}^{2}\right)^{2}}-\Delta_{I_{2}}>\frac{B}{3}-\frac{B^{2}}{2 k \bar{\varepsilon}^{2}} \quad \text { (A37) } \\
\frac{1}{8} B \frac{4 B^{2}\left(1-\gamma_{I_{1}}\right)^{2}+B k \bar{\varepsilon}^{2}\left(7 \gamma_{I_{1}}-8\right)+2 k^{2} \bar{\varepsilon}^{4}}{\left(B \gamma_{I_{1}}-B+k \bar{\varepsilon}^{2}\right)^{2}}>\frac{1}{2} B k \bar{\varepsilon}^{2} \frac{k \bar{\varepsilon}^{2}-B}{\left(B \gamma_{I_{2}}-B+k \bar{\varepsilon}^{2}\right)^{2}}
\end{gathered}
$$

$$
\begin{gathered}
\frac{1}{8} B \frac{4 B^{2}\left(1-\gamma_{I_{1}}\right)^{2}+B k \bar{\varepsilon}^{2}\left(7 \gamma_{I_{1}}-8\right)+2 k^{2} \bar{\varepsilon}^{4}}{\left(B \gamma_{I_{1}}-B+k \bar{\varepsilon}^{2}\right)^{2}}-\Delta_{I_{1}} \\
>\frac{1}{8} B \frac{4 B^{2}\left(1-\gamma_{I_{2}}\right)^{2}+B k \bar{\varepsilon}^{2}\left(7 \gamma_{I_{2}}-8\right)+2 k^{2} \bar{\varepsilon}^{4}}{\left(B \gamma_{I_{2}}-B+k \bar{\varepsilon}^{2}\right)^{2}}-\Delta_{I_{2}} \\
\gamma_{I_{2}}>\gamma_{I_{1}}>1,
\end{gathered}
$$

with $\Delta_{I_{1}}, \Delta_{I_{2}}>0$. In Proposition 1(i) and Proposition 3(i) the crucial condition that $E$ accepts $I_{1}$ 's offer could not be met. Here, (A39') shows that this condition holds as long as $\Delta_{I_{1}}$ is sufficiently small and $\Delta_{I_{2}}$ is sufficiently large. Altogether, there are feasible parameter constellations for which $\left(\mathrm{A} 37^{\prime}\right)-\left(\mathrm{A} 40^{\prime}\right)$ hold at the same time. ${ }^{28}$

Next, (ii) is considered. A mismatch II equilibrium will exist if

$$
\begin{gathered}
E U_{I_{2}}^{-}\left(e_{I_{2}}^{*} ; s_{2}\right)<E U_{i}\left(e_{i}^{*} ; s_{1}\right), \quad i \in\left\{I_{1}, I_{2}, E\right\} \\
E U_{I_{1}}^{-}\left(e_{I_{1}}^{*} ; s_{3}\right)>E U_{i}\left(e_{i}^{*} ; s_{1}\right), \quad i \in\left\{I_{1}, I_{2}, E\right\}
\end{gathered}
$$

\footnotetext{
${ }^{27}$ Condition (A36) becomes irrelevant, because it is already fulfilled as (A37), or (A41), holds.

${ }^{28}$ This can easily be checked by a numerical example; let, e.g., $k=1, \bar{\varepsilon}=1, B=1$, $\gamma_{I_{2}}=10, \gamma_{I_{1}}=9.5, \Delta_{I_{2}}=0.5, \Delta_{I_{1}}=0.1$.
} 


$$
\begin{gathered}
E_{\hat{\gamma}_{I_{1}}}\left[E U_{E}^{-}\left(e_{E}^{*} ; s_{3}\right)\right]>E U_{i}\left(e_{i}^{*} ; s_{1}\right), \quad i \in\left\{I_{1}, I_{2}, E\right\} \\
\gamma_{I_{2}}<\gamma_{I_{1}}<1 .
\end{gathered}
$$

From the proof of Proposition 3 we know that $E U_{E}^{-}\left(e_{E}^{*} ; s_{3}\right) \equiv E U_{I_{1}}^{-}\left(e_{I_{1}}^{*} ; s_{3}\right)$ which is either convex or concave in $\gamma_{I_{1}}$ for different ranges of $\gamma_{I_{1}}$. If convexity holds, inequality (A42) implies condition (A43) to hold. Then, we have the same conditions compared to (A5)-(A7) with symmetric information. If concavity holds, inequality (A42) will become irrelevant, because (A43) is stronger. Then, the conditions for a mismatch II equilibrium are less likely to be met than the conditions (A5) - (A7) for mismatch II under symmetric information.

The result of Proposition 4(iii) immediately follows from the fact that $I_{1}$ and $I_{2}$ have the same information as in Proposition 1. Therefore, a mismatch III is as likely as in the situation with symmetric information.

\section{References}

Holt, Charles A., and Roger Sherman, 1994. The loser's curse. American Economic Review, 84, 642-652.

Jovanovic, Boyan, 1984. Matching, turnover, and unemployment. Journal of Political Economy, 92, 108-122.

Kräkel, Matthias, 1999, Strategic mismatches in sequential contracting: the case of professional partnerships. Discussion Paper No. A-604. University of Bonn. Department of Economics.

Lazear, Edward P., 1989, Pay equality and industrial politics. Journal of Political Economy, 97, 561-580. 
Lazear, Edward P., 1995, Personnel economics (Cambridge/Mass.).

Lazear, Edward P., and Sherwin Rosen, 1981, Rank-order tournaments as optimum labor contracts. Journal of Political Economy, 89, 841-864.

Milgrom, Paul R., 1981. Good news and bad news: representation theorems and applications. Bell Journal of Economics, 12, 380-391.

Milgrom, Paul R., 1985, The economics of competitive bidding: a selective survey, In: Leonid Hurwicz, David Schmeidler, and Hugo Sonnenschein, (Eds.), Social goals and social organization. Cambridge, 261-289.

Milgrom, Paul R., and Sharon Oster, 1987, Job discrimination, market forces, and the invisibility hypothesis. Quarterly Journal of Economics, $102,453-476$.

Mood, Alexander M., Graybill, Franklin A., and Duane C. Boes, 1974, Introduction to the theory of statistics. third edition (Singapore).

Mortensen, Dale T., 1988. Wages, separations, and job tenure: on-thejob specific training or matching? Journal of Labor Economics, 6, $445-471$.

Nalebuff, Barry J., and Joseph E. Stiglitz, 1983, Prizes and incentives: toward a general theory of compensation and competition. Bell Journal of Economics, 14, 21-43.

O'Malley, Martin, and Sean O'Malley, 1994, Game day - the Blue Jays at sky dome (Toronto).

Roll, Richard, 1986, The hubris hypothesis of corporate takeovers. Journal of Business, 59, 197-216. 


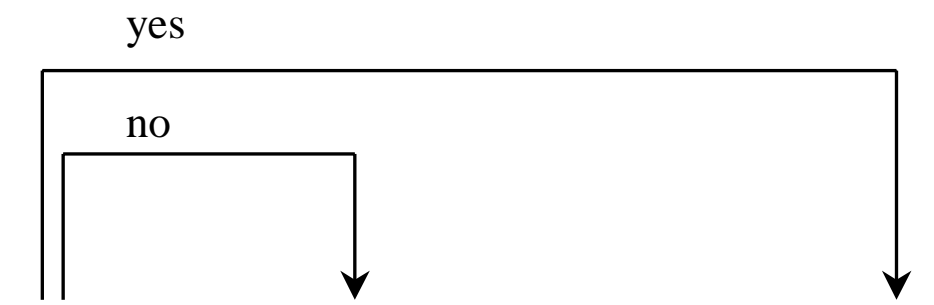

$I_{1}$ decides about $\quad$ E accepts $I_{2}$ decides about $\quad$ E accepts

contract offer or rejects contract offer or rejects tournament

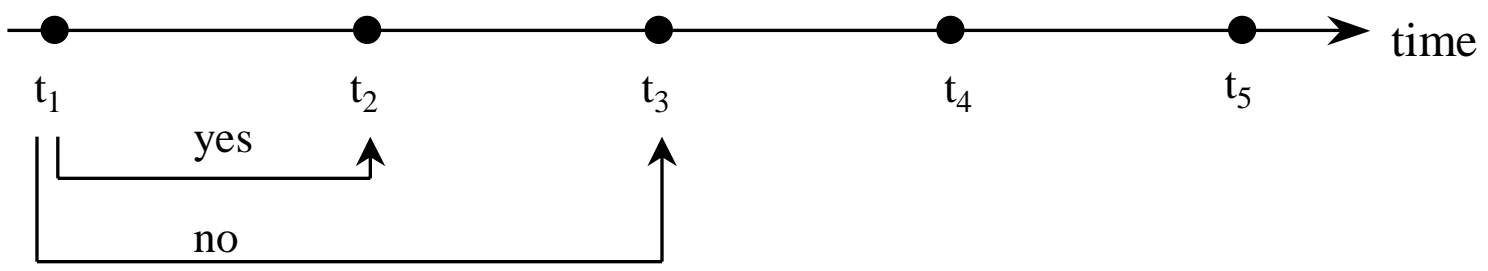

Figure 1: Timing of the sequential moves 


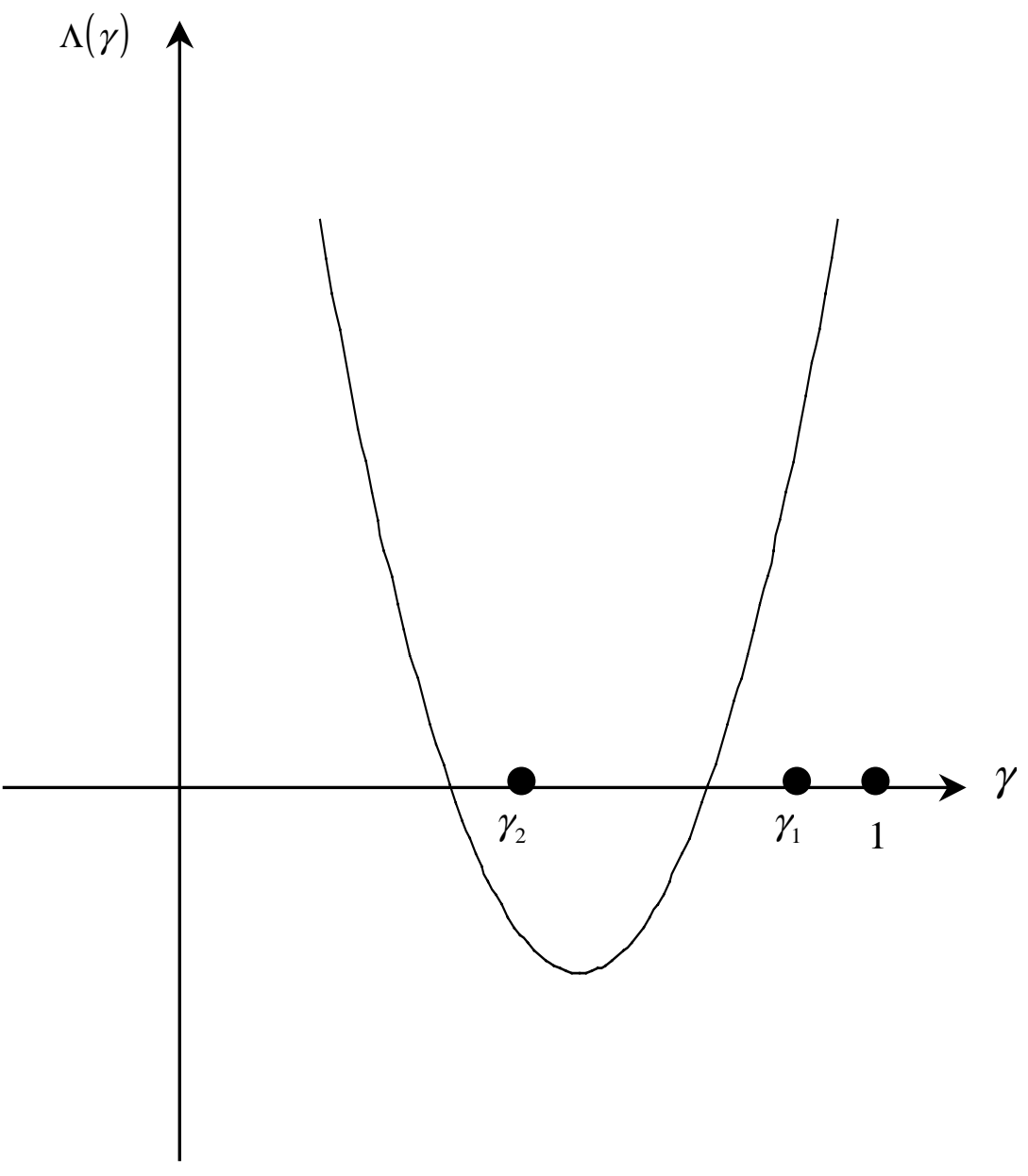

(a) $\Lambda(\gamma)$ is convex

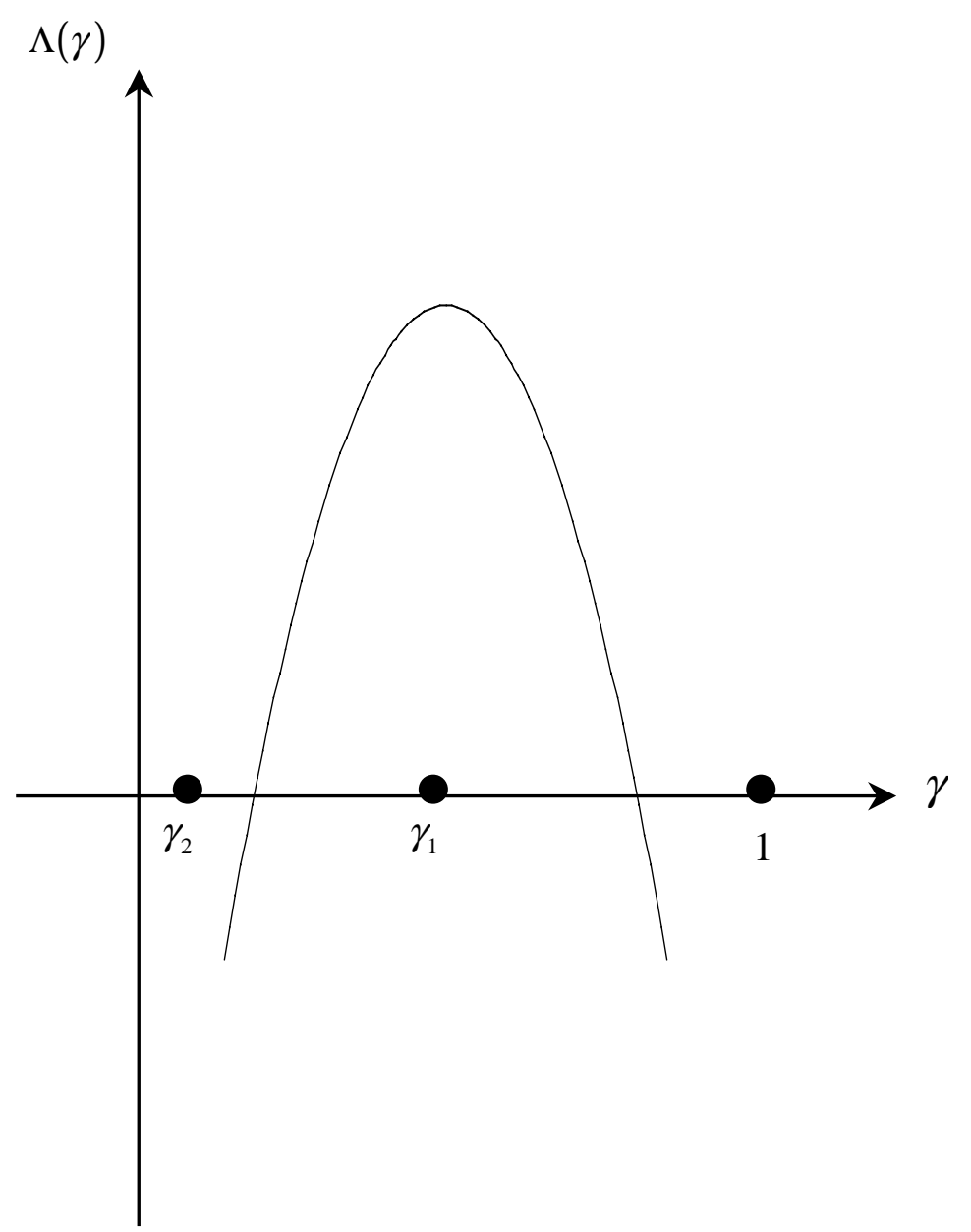

(b) $\quad \Lambda(\gamma)$ is concave

Figure 2: Parabola $\Lambda(\gamma)$ (in (a) the left-hand root of $\Lambda(\gamma)$ is allowed to be negative; in (b) the right-hand root of $\Lambda(\gamma)$ is allowed to be greater than one) 